\title{
STABLE THICKENINGS IN THE HOMOTOPY CATEGORY $\left({ }^{\mathbf{1}}\right)$
}

\author{
BY
}

\author{
R. L. CHAZIN( $\left.{ }^{2}\right)$
}

\begin{abstract}
This paper extends the result that the set of stable thickenings of a simplyconnected complex $K$ are in 1-1 correspondence with $[K, B Q](Q=\mathrm{O}, \mathrm{PL}$, or TOP). which holds in the smooth, PL, and topological categories, to the homotopy category.
\end{abstract}

In this paper we consider the notion of thickening (introduced by Mazur and Wall [14]) in the homotopy category. The main result is the following:

Theorem 1. Let $K$ be a finite, pointed, 2-connected Poincaré complex. Then there is a natural map $\tau: \supset(K) \rightarrow[K, B G]$ which is a bijection.

Here $\neg(K)$ denotes the set of stable thickenings of $K$, and $B G=\lim B G_{n}$, where $G_{n}$ is the group of homotopy equivalences of $S^{n-1}$. As is well known [12], $[K, B G]$ is in 1-1 correspondence with stable fibre-homotopy equivalence classes of (stable) spherical fibre spaces over $K$.

A theorem of this kind has been established in the differentiable category by Mazur, in the PL category by Wall [14] and in the topological category by the author [5].

It is probable that a theorem of this kind holds without the connectivity hypothesis. Such a theorem would follow, for instance, if we had a nonsimply connected version of Browder's theorem [2, 1.4.1] that the inclusion $A \subset X$ of a Poincaré pair $(X, A)$ is a spherical fibration. $\left({ }^{3}\right)$

1. Preliminaries. We work in the homotopy category of pointed topological spaces and homotopy classes of base-point preserving maps.

Let $K$ be a finite, simply-connected $\mathrm{CW}$ complex of dimension $k$ with basepoint *, and $(M, \partial M)$ a Poincaré pair of dimension $m$, and base-point $* \in \partial M .\left(^{4}\right)$ Then a (Poincaré complex-, or homotopy-) prethickening of $K$ will be a base-point

Received by the editors March 21, 1973.

AMS (MOS) subject classifications (1970). Primary 57B10, 57F10.

Key words and phrases. Poincaré complex, homotopy category, thickening, stable thickening, spherical fibration.

(1) The author wishes to dedicate this work to the memory of his father, Benjamin J. Chazin (July 8, 1901-September 29, 1972).

(2) Partially supported by National Science Foundation Grant GP-7913.

(3) Since this was written, such results have been obtained by Browder [3] and the author [4].

(4) For definitions see [15]. Slightly different definitions are given in [6]. [6] and [7] discuss other questions on homotopy thickenings. 
preserving homotopy equivalence $\varphi: K \rightarrow(M, \partial M)$, where $m \geq k+3$. We will denote the codimension of $K$ in $M$ by $n=m-k$.

Two prethickenings $\left(M_{1}, \varphi_{1}\right)$ and $\left(M_{2}, \varphi_{2}\right)$ will be said to be equivalent if there is a homotopy equivalence of pairs $h:\left(M_{1}, \partial M_{1}\right) \rightarrow\left(M_{2}, \partial M_{2}\right)$ such that the following diagram commutes:

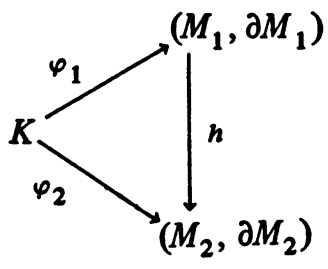

A Poincaré complex, or homotopy $m$-thickening, or briefly, a thickening, of $K$ will then be an equivalence class of prethickenings. In this paper we work only in the homotopy category and all thickenings will be Poincare complex or homotopy thickenings unless otherwise stated.

This definition is slightly different from Wall's definition for the smooth or PL cases as we have imposed the requirement of simple connectivity. This is made necessary by the lack of a suitable embedding theorem for non-simply-connected Poincaré complexes.

We now consider splittings and embeddings of Poincaré complexes. A splitting of a Poincaré complex $M^{n}$ is a pair of Poincaré complexes $\left(N_{1}^{n}, \partial N_{1}^{n}\right),\left(N_{2}^{n}, \partial N_{2}^{n}\right)$ such that $\partial N_{1}=\partial N_{2}=N_{1} \cap N_{2}$ and a homotopy equivalence $\varphi: M \rightarrow N_{1} \cup N_{2}$. This idea extends in the obvious way to splittings of Poincare pairs.

Following [8], let $K^{k}$ be a CW complex and $M^{n}$ a Poincaré complex, and let $f: K^{k} \rightarrow M^{n}$. Then $f$ is said to be homotopic to an embedding if there is a splitting $\left\{\left(N_{1}^{n}, \partial N_{1}^{n}\right),\left(N_{2}^{n}, \partial N_{2}^{n}\right), \varphi\right\}$ and a homotopy equivalence $h: K^{k} \rightarrow N_{1}^{n}$ such that

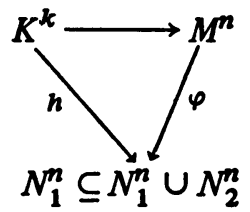

is homotopy commutative, and such that the inclusion $\partial N_{1}^{n} \subseteq N_{1}^{n}$ is $(n-k-1)$ connected. For details, see $[9, \S 2]$, or $[15$, Chapter 11]. Levitt $[8,3.1]$ gives the following embedding theorem, with the usual restrictions on Poincare complexes (which we shall omit, preferring, as in [10], to regard them as part of the definition):

Theorem 2 (Levitt). Let $\left(M^{n}, \partial M^{n}\right)$ be a simply-connected Poincaré pair, $K^{k}$ a $C W$ complex, $n \geq 2 k+2$, and $f: K^{k} \rightarrow M^{n}$. Then $f$ is homotopic to an embedding.

By using this theorem, we can construct an induced map on thickenings:

Proposition 3. Let $K^{k}, L^{l}$ be finite, pointed, simply-connected $C W$ complexes and 
$f: K^{k} \rightarrow L^{l}$ a map. If $m \geq 2 k 2,2 l+2$, then $f$ induces $f^{*}: \square^{m}\left(L^{l}\right) \rightarrow \square^{m}\left(K^{k}\right)$.

Proof. Let $(M, \varphi)$ be a thickening of $L$; then $\varphi f: K \rightarrow M$. Applying Levitt's theorem to $\varphi f$, we obtain as part of the resulting splitting of $\left(M^{n}, \partial M^{n}\right)$ a Poincaré complex $\left(N_{1}, \partial N_{1}\right)$ and a homotopy equivalence $h: K \rightarrow\left(N_{1}, \partial N_{1}\right)$ so that the following diagram is homotopy commutative:

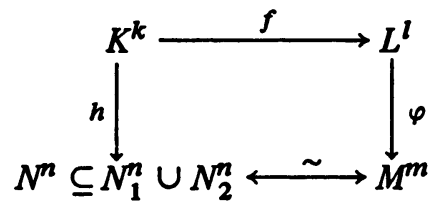

Then $\left(N_{1}, h\right)$ is the required thickening of $K$.

Although the preceding construction requires a severe restriction on the dimension of $M$, this will not cause us any difficulty since we will be working in the stable range.

Now let $f: K^{k} \rightarrow * . f$ induces $f^{*}: \beth^{m}(*) \rightarrow \beth^{n}(K) . D^{m} \in \beth^{m}(*)$; by the trivial $m$-thickening we shall mean $f^{*}\left(D^{m}\right)$. We denote this thickening by $K_{0}$.

Note that if $f: K^{k} \rightarrow L^{l}$ is a homotopy equivalence and $m \geq 2 l+2,2 m+2$, then $f^{*}: \beth^{m}\left(L^{l}\right) \rightarrow \beth^{\prime}\left(K^{l}\right)$ is a bijection. In particular $f^{*}: \beth^{m}\left(K^{k}\right) \rightarrow \beth^{m}\left(K_{0}\right)$ for $m$ sufficiently large.

Stable thickenings. As usual we have the suspension map

$$
\cdots \rightarrow \beth^{m}(K) \stackrel{\Sigma^{m}}{\longrightarrow} \square^{m+1}(K) \rightarrow \cdots .
$$

By Levitt [8], $\Sigma^{m}$ is an isomorphism for $m \geq k+2$. We let $\beth(K)$ denote the common stable value; $\supset(K)$ will be called the set of stable homotopy thickenings of $K$.

Note that in establishing the existence of $\supset(K)$, Levitt's result enables us to dispense with a homotopy analogue of Wall's suspension sequence $[1, \S 5]$. No such analogue exists at the present time and it would be interesting to determine if one does, indeed, exist.

2. Proof of Theorem 1. We now define maps $\Psi, \Phi$ as follows, with

$$
\sqsupset(K) \underset{\Phi}{\stackrel{\Psi}{\rightleftarrows}}[K, B G]
$$

To define $\Psi$, let $\left(M^{m}, \gamma\right)$ be a prethickening of $K$. As $\left(M^{m}, \partial M^{m}\right)$ is a Poincaré pair, there is a unique (up to stable fibre-homotopy equivalence) Spivak normal fibration $\nu$ and hence a classifying map $c_{\nu}: M \rightarrow B G$, unique up to homotopy. Furthermore, as is well known, $[K, B G]$ is a group, so inverses are defined, and $-c_{v}=c_{-v}$. Define $\Psi([M, \gamma])$ to be the homotopy class of the map $c_{-, \gamma} \gamma$ $\in[K, B G] . \Psi$ is well defined since varying $(M, \gamma)$ by an equivalence only varies $\gamma c_{-}$, by a homotopy, so that $\Psi$ is well defined.

To define $\Phi$, let $\left(K_{0}, \alpha\right)$ be a trivial thickening of $K$ and $\beta$ a homotopy inverse 
for $\alpha$. Let $f=f_{\xi} \in\left[f_{\xi}\right] \in[K, B G]$ be associated to the spherical fibration $\xi$ over $K$. Form the associated "disc fibration" $\bar{\xi}$ over $K$ by "coning each fibre" [1, Appendix]. This is a construction analogous to forming the disc bundle associated to a sphere bundle. Let $\mu$ be the zero section; $\left(E_{\bar{\xi}}, \mu\right)$ is then a thickening of $K$, and we define $\Phi\left(\left[f_{\xi}\right]\right)=\left[\left(E_{\xi}, \mu\right)\right]$. Note that using $\beta$ we also obtain a thickening of $K_{0}$ by forming $\left(E^{*}, \mu^{*}\right)=\beta^{*}\left(E_{\xi}, \mu\right)$. This will figure in the sequel. $\Phi$ is well defined, since if we vary $f_{\xi}$ by a homotopy, $\bar{\xi}$ varies by a fibre homotopy equivalence mod boundaries and hence we obtain equivalent thickenings.

Note that since $K$ and $K_{0}$ have the same homotopy type, two fibrations $\eta_{1}, \eta_{2}$ are fibre-homotopy equivalent over $K$ iff their induced fibrations $\beta^{*} \eta_{1}, \beta^{*} \eta_{2}$ are fibre-homotopy equivalent over $K_{0}$.

For the proof of the theorem, we first prove that $\Phi \Psi=1$. By analogy with the differentiable case we have:

Lemma 4. Let $(X, Y)$ be a Poincaré n-pair, and $\nu$ the normal fibration of $X$, $\nu^{\prime}$ the normal fibration of $Y$. Then if $i: Y \subset X$,

$$
i^{*} \nu \sim_{s}\left(\left(i^{*} \nu\right) * 1\right) \sim_{s} \nu^{\prime}
$$

Remark. Here * also indicates join, and $\sim_{s}$ denotes stable fibre homotopy equivalence.

Proof. The first relation follows by stability. We use Browder's version of Wall's statement of the uniqueness of the Spivak normal fibration, according to which, given a Poincaré $n$-pair $(X, Y)$, we obtain a normal $(k-1)$ spherical fibration $\nu$ over $X$ and an element $\alpha \in \pi_{n+k}(T(\nu), T(\nu \mid Y))$ such that $h(\alpha) \cap U=[X] \in H_{n}(X, Y)$, where $h$ is the Hurewicz homomorphism, $U$ is the Thom class of $\nu$ and $T$ denotes the Thom space. Then $v$ is unique up to some fibre homotopy equivalence $b$, and $T(b)$ sends $\alpha_{1}$ onto $\alpha_{2}$. (See [2, I.4.19] for details.) The desired result then follows by checking that commutativity holds in the diagram:

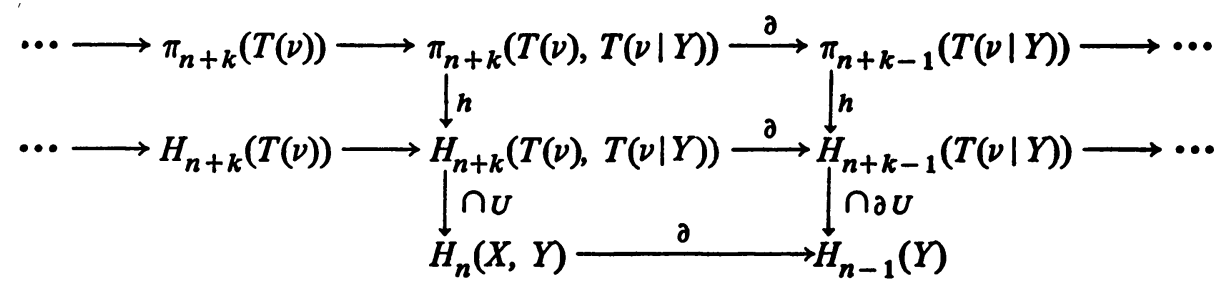

Note that in the bottom row $[X] \rightarrow[Y]$. Since $\nu \mid Y$ and $\nu^{\prime}$ both satisfy

$$
h(\xi) \cap U=[Y] \in H_{n-1}(Y),
$$

the assertion follows from the uniqueness theorem. We now apply the result of Sutherland [13]:

Proposition 5 (Sutherland). Let $M$ be a finite 1-connected $C W$ complex over 
which there is a reducible sphere fibering $\nu$. Let $\pi: B \rightarrow M$ be the projection of a $q$ sphere fibering $\xi(q>1)$ and let $\gamma$ be a negative for $\xi$. Then the sphere fibering $\pi^{*}(\gamma * \nu * 1)$ over $B$ is reducible.

Proof. By the original uniqueness theorem of Spivak, this means that $\pi^{*}(\eta * \nu * 1)$ is the normal fibration of $B$. We apply this to the fibration $E^{*}=\beta^{*} E_{\bar{\xi}}$ over $K_{0}$ defined above. Let $\pi$ be the projection of $E^{*}$. Sutherland's result then asserts that $\nu\left(\partial E^{*}\right)$ is $\pi^{*}\left(\eta * \nu\left(K_{0}\right) * 1\right)$, where $\eta$ is an inverse for $\partial E^{*} \rightarrow K_{0}$, and $\nu\left(K_{0}\right)$ is the normal fibration of $K_{0}$, which is trivial. Computing $\Phi\left(\nu\left(E^{*}\right)\right)$ (and hence $\Phi\left(E_{\xi}\right)$ ) amounts to computing the classifying map of $-\nu\left(E^{*}\right)$. Since 1 and $\nu\left(K_{0}\right)$ are trivial, and using Lemma 4, this is equivalent to $c_{-\eta}$, which is homotopic to $c_{\xi}$, and we have the following commutative diagram:

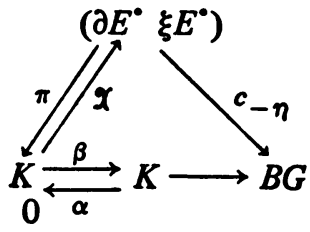

where $\mathcal{X}$ is a zero section for $E^{*} \rightarrow K_{0}$.

Thus $\Phi \Psi=1, \Phi$ is $1-1$ and $\Psi$ is onto.

It remains to prove, for instance, that $\Phi$ is onto. Let $\left(M^{m}, \gamma\right)$ be a thickening of $K$. Using the path fibration $c$ of $[11,2.8 .9]$ (relativized) we can replace $\left(M^{m}, \partial M^{m}\right)$ by a homotopically equivalent pair $\left(\left(M^{\prime m}, \partial M^{\prime m}\right), \gamma^{\prime}\right)$, which is a thickening, and such that the homotopy inverse $\pi^{\prime}$ of $\gamma^{\prime}$ is a fibration. Thus we may assume that $\left(M^{m}, \partial M^{m}\right)$ itself is a fibration; we have

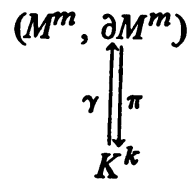

Let $(F, \partial F)$ be the relative fibre. Applying the homotopy sequence of the fibration

$$
\cdots \rightarrow \pi_{n}\left(M^{m}\right) \rightarrow \pi_{n}(K) \rightarrow \pi_{n-1}(F) \rightarrow \pi_{n-1}\left(M^{m}\right) \rightarrow \pi_{n-1}(K) \rightarrow \cdots
$$

we find that all homotopy groups by $F$ vanish and hence that $F$ is contractible.

It remains to show that $\partial F=F \cap \partial M$ is actually a homotopy $S^{n-1}$ $(m=n+k)$. We do this using a relative Serre spectral sequence with

$$
E_{p, q}^{2}=H_{p}\left(K, H_{q}(F, \partial F)\right)
$$

and $E^{\infty}=$ subquotients of $H_{n}(M, \partial M)$. We must show that 


$$
\begin{array}{rlrl}
H_{j}(F, \partial F) & =0, & j \neq n, \\
& =Z, & & j=n .
\end{array}
$$

Using the exact homology sequence of $(F, \partial F)$ it then follows that $\partial F$ is a homology $S^{n-1}$.

Lemma 6. $H_{j}(F, \partial F)=0, j<n$.

Proof. $K$ is simply connected and $\operatorname{dim} K=k$; so

$$
H_{j}(M, \partial M) \approx H^{n+k-j}(M)=H^{n+k-j}(K) \approx 0, \text { if } j<n .
$$

Let $s$ be the first index $>0$ such that $H_{s}(F, \partial F) \neq 0$. Then

$$
E_{0, q}^{2}=H_{0}\left(K, H_{q}(F, \partial F)\right) \cong H_{q}(F, \partial F)
$$

(coefficients in a field). For the differentials we have, with $p=0$,

$$
E_{r, q-r+1}^{r} \stackrel{d^{r}}{\rightarrow} E_{0, q}^{r} \stackrel{d^{r}}{\rightarrow} E_{-r, q+r-1}^{r} .
$$

The right-hand group is always zero, as $E$ is a first quadrant spectral sequence. For $q \leq s, E_{r, q-r+1}^{2}$ is zero; hence $E_{r, q-r+1}^{r}$ is 0 for $r>1$, and all differentials vanish, so that $E_{0, q}^{r} \cong E_{0, q}^{\infty}$ In particular, for $q=s$ we obtain $0 \neq E_{0, s}^{\infty}$ $\subseteq H_{s}(M, \partial M)$ and the latter group is zero for $s<n$, a contradiction.

Next consider $H_{n}(F, \partial F)$. We have $E_{0, n}^{2}=H_{n}(F, \partial F)$, and differentials

$$
E_{r, n-r+1}^{r} \stackrel{d^{r}}{\longrightarrow} E_{0, n}^{r} \stackrel{d^{r}}{\longrightarrow} E_{-r, n+r-1}^{r} .
$$

As before, the right-hand group is always zero. For the left-hand group we look at $E_{r, n-r+1}^{2}$. This is always 0 for $r \neq n+1$, since in that case $H_{n-r+1}(F, \partial F)=0$. For $r=n+1$ we have

$$
\begin{aligned}
E_{n+1,0}^{2} & =H_{n+1}\left(K, H_{0}(F, \partial F)\right) \\
& =0, \text { since } n+1 \gg \operatorname{dim} K .
\end{aligned}
$$

Hence all differentials vanish and

$$
H_{n}(M, \partial M)=E_{0, n}^{\infty} \cong E_{0, n}^{2} \cong H_{n}(F, \partial F) .
$$

But $H_{n}(M, \partial M) \cong H^{k}(K)=Z$. Hence $H_{n}(F, \partial F)=Z$ and $H_{n-1}(\partial F)=Z$ so that $\partial F$ is a homology $(n-1)$-sphere.

Finally, $H_{i}(F, \partial F)=0$ for $i>n$. For, by finite dimensionality, $(F, \partial F)$ has a highest dimensional nonzero homology group, say, in dimension $q$. Now consider the spectral sequence with $E_{k+2, q-1}^{2} \rightarrow E_{k, q}^{2} \rightarrow E_{k-2, q+1}^{2}$. The right-hand group is $H_{k-2}\left(K, H_{q+1}(F, \partial F)\right)$ and hence is zero; the left-hand group is $H_{k+2}\left(K, H_{q}(F\right.$, $\partial F)$ ) and is zero as $K$ is $k$-dimensional. Hence, the middle term survives to $E^{\infty}$, 
and gives a nonzero summand for the right side of the equation

$$
\operatorname{dim} H_{r}(M, \partial M)=\sum E_{p, q}^{\infty}
$$

where the summation is carried out for $p+q=r$. Since by definition of $q$, $k+q>m$, we get the required contradiction.

It remains to prove that $\partial F$ is simply connected; it will then follow from the Whitehead theorem that $\partial F$ is a homotopy $(n-1)$-sphere.

Consider the homotopy ladder of the fibration

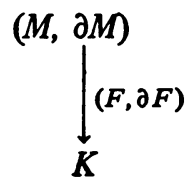

We obtain

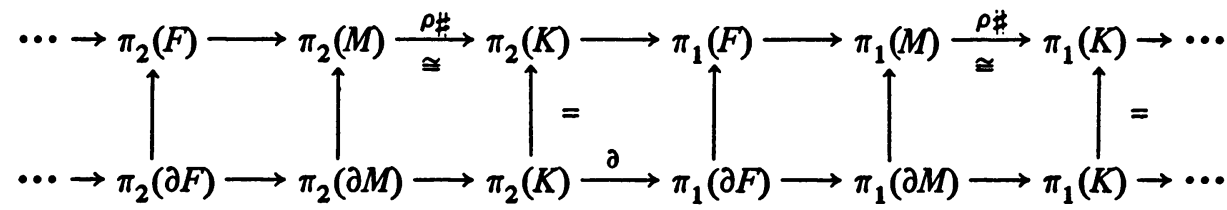

By hypothesis, we have $\pi_{1}(\partial M)=\pi_{1}(K)=0$; so $\partial$ is onto. Then $\pi_{2}(K)=0$ gives $\pi_{1}(\partial F)=0$. This completes the proof.

\section{REFERENCES}

1. W. Browder,The Kervaire invariant of framed manifolds and its generalization, Ann. of Math. (2) 90 (1969), 157-186. MR 40 \#4963.

2.—, Surgery on simply connected manifolds, Notes, Princeton University, Princeton, N.J., 1969.

3._- Poincaré spaces, their normal fibrations, and surgery (preprint).

4. R. L. Chazin, Normal fibrations for Poincaré complexes (preprint).

5. - Stable thickenings in the topological category, Proc. Amer. Math. Soc. 29 (1971), 175-178.

6. J. P. E. Hodgson, Poincaré complex thickenings and concordance obstructions, Bull. Amer. Math. Soc. 76 (1970), 1039-1043. MR 41 \#9263.

7.—_, Exact sequences and concordance obstructions. I, II (to appear).

8. N. Levitt, On the structure of Poincaré duality spaces, Topology 7 (1968), 369-388. MR 40 \#2089.

9. - Normal fibrations for complexes, Illinois J. Math 14 (1970), 385-408. MR 43 \# 1198.

10. M. Spivak, Spaces satisfying Poincaré duality, Topology 6 (1967), 77-101. MR 35 \#4923.

11. E. H. Spanier, Algebraic topology, McGraw-Hill, New York, 1966. MR 35 \# 1007.

12. J. Stasheff, A classification theorem for fibre spaces, Topology 2 (1963), 239-246. MR 27 \#4235.

13. W. A. Sutherland, Homotopy-smooth sphere fibrings, Bol. Soc. Mat. Mexicana (2) 10 (1965), 73-79. MR 35 \# 7342.

14. C. T. C. Wall, Classification problems in differential topology. IV. Thickenings, Topology 5 (1966), 73-94. MR 33 \# 734.

15. - Surgery on compact manifolds, Academic Press, New York, 1971.

Department of Mathematics, University of California at Irvine, Irvine, California 